to

Department of Energy

Pittsburgh Energy Technology Center

P. O. Box 10940

Pittsburgh, PA 15236

\title{
PELletization OF FINE COALS
}

(DOE Grant No. DE-FG-22-89PC89766)

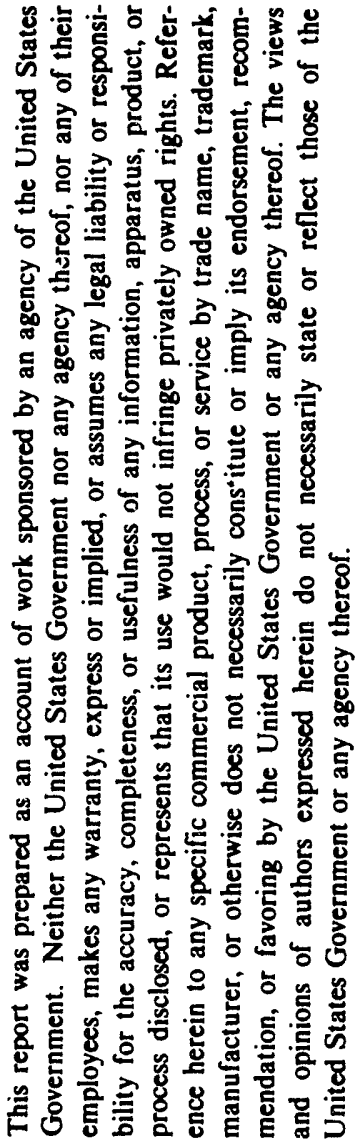

Reporting Period

March 1, 1991 to May 30, 1991

Principal Investigator: Kal V. S. Sastry

Phone: 415-642-3808

University of California

College of Engineering

Department of Materials Science

and Mineral Engineering

Berkeley, CA 94720

September 1991 


\section{Project Overview}

Environmental problems associated with acid rain are attributed to sulfur emissions from coal-fired utility boilers. Sulfur contents of 2 to 6 percent in coals from Eastern and Midwestern states are considered to be responsible for the bulk of the US sulfur emissions. A significant portion of total sulfur can be removed prior to combustion through such low-cost generic coal cleaning techniques as advanced froth flotation and oil agglomeration. In addition to the environmental concerns, demands for low-ash, low-sulfur coals and economic constraints for high productivity are requiring the coal industry to use modern mining methods such as longwall mining. Also, better utilization of coals is attempted through synthetic fuel production. All these technologies are faced with one common problem - fine coals. Dealing effectively with these fine coals during handling, storage, transportation, and/or processing continues to be a challenge facing the industry. Agglomeration, the unit operation of producing larger bodies from fine powders, alleviates many of the problems associated with fine particulates. For example, some of the benefits provided by pelletizing coal fines include improved fugitive dust control, decreased transportation losses, reduced risk of coal freezing, and lowered risk of spontaneous combustion. Only a partial understanding of the fundamentals of coal pelletization exists today.

The present research project attempts to provide a basis to determine the pelletizability of fine coals, to ascertain the role of additives and binders and to establish a basis for binder selection. Currently, there are no established techniques for determining the quality of coal pellets. Our research is intended to develop a series of tests on coal pellets to measure their storage characteristics, transportability, ease of gasification and rate of combustion. Information developed from this research should be valuable for making knowledgeable decisions for on-time plant design, occasional binder selection and frequent process control during the pelletization of coal fines. 


\section{Progress During Last Quarter}

During the last quarter, we continued the batch pelletization studies on Upper Freeport coal. The results as presented in that last quarterly report (April 1991) indicated that the surface conditions on the coal particle influenced the pelletizing growth rates. For example, a fresh (run of mine) sample of coal will display different pelletizing growth kinetics than a weathered sample of the same coal. Since coal is a heterogeneous material, the oxidized product of coal is equally variable. We found it to be logistically difficult to consistently produce large quantities of artificially oxidized coal for experimental purposes and as such we have used a naturally weathered coal. We have plans to oxidize coals under controlled oxidizing conditions and be able to establish their pelletizing behavior.

The next phase of experiments were directed to study the effect of surface modification, introduced during the coal cleaning steps, on pelletizing kinetics. Accordingly, we initiated studies with two additives commonly used during the flotation of coal: dextrin (coai depressant) and dodecane (coal collector).

Dextrin is a modified starch and when added to a coal slurry, it acts as a depressant. Dextrin has also been used extensively in froth flotation studies at U.C. Berkeley. The flotation tests have indicated that Upper Freeport coal responds strongly to dextrin. We suspected that pelletization tests with Upper Freeport coal and dextrin might show equally strong responses.

Figure 1 (which was originally presented in the March 1991 report) shows the pelletization of behavior of as-received Upper Freeport coal fines with different addition of moisture contents. Like most coals, this coal could be pelletized only over a narrow range of moisture additions from 25 to 32 percent by weight (dry basis). With lower moisture addition, the Upper Freeport coal demonstrates a long induction period followed by a rapid, clumping type of growth. With high moisture addition, a much shorter induction period was observed. The product pellets were spherical, but were very moist on the surface. 
During the next series of experiments we studied the influence of small additions of dextrin with low moisture addition of 26 percent. Figure 2 presents the growth curves for various amounts of added dextrin to Upper Freeport coal. The apparent effect of dextrin is to act as a "accelerating" agent - that is, the growth curve is shifted such that larger pellets are produced within a given period of time. The experiments suggest that a minimum amount of dextrin must be added to affect the growth curve. For our experiments with Upper Freeport coal, this threshold amount of dextrin is about 0.125 percent by dry weight. There is also an apparent upper limit on how much dextrin to add. The curves suggest that adding dextrin in quantities greater than 0.25 percent (for example as much as 1.00 percent) has no additional effect.

Next, we conducted experiments on two other bituminous coal samples (Pittsburgh No. 8, and Illinois No. 6) to determine whether dextrin has a similar effect. First, we developed the pelletization curves for each coals with varying amounts of moisture only. Next, we added small quantities of dextrin to each of the coal. Figures 3 and 4 compare the growth curves with and without dextrin. These plots show that dextrin does influence the growth curves of Pittsburgh No. 8 and Illinois No. 6 coals. Based on the results from these three coal samples, we arrive at a tentative conclusion that the addition of dextrin causing the coal surface to becoming hydrophilic accelerates the pellet growth rate.

We subsequently ran pelletizing experiments with the addition of dextrin to anthracite fines. The results are presented in Figure 5. These results indicate that dextrin does not have the same influence on anthracite as it did have on the bituminous coals. This effect must be due to the fact that dextrin does not make the surface of anthracite hydrophilic to a substantial extent.

For the next quarter, our plans are to continue our studies on the influence of dodecane on pelletization. We also plan to design additional experiments to give us an understanding of the mechanisms of the interaction between surface-active reagents and the coal surface. 


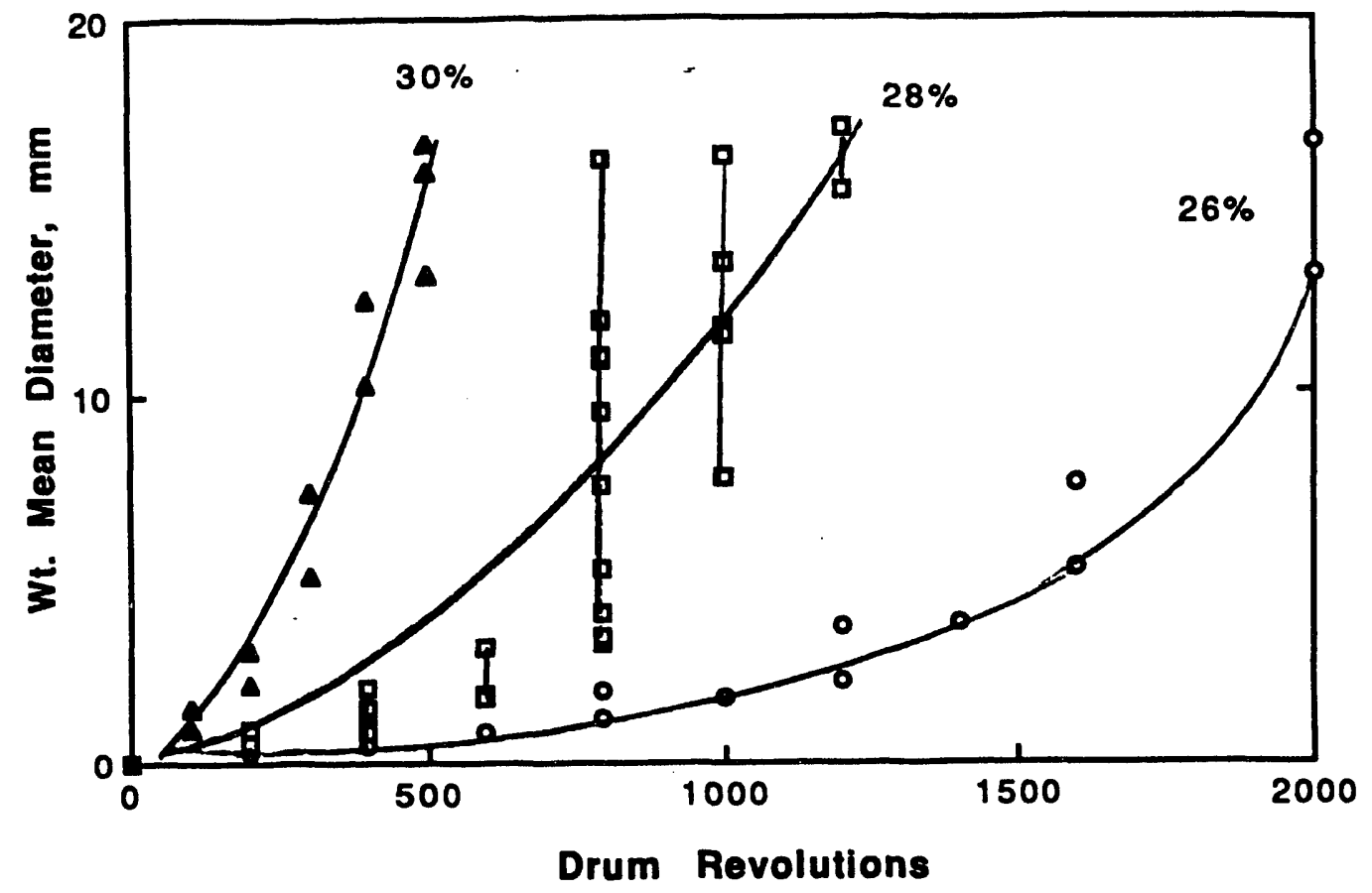

Figure 1. Influence of feed moisture on the batch pelletization kinetics of Upper Freeport coal.

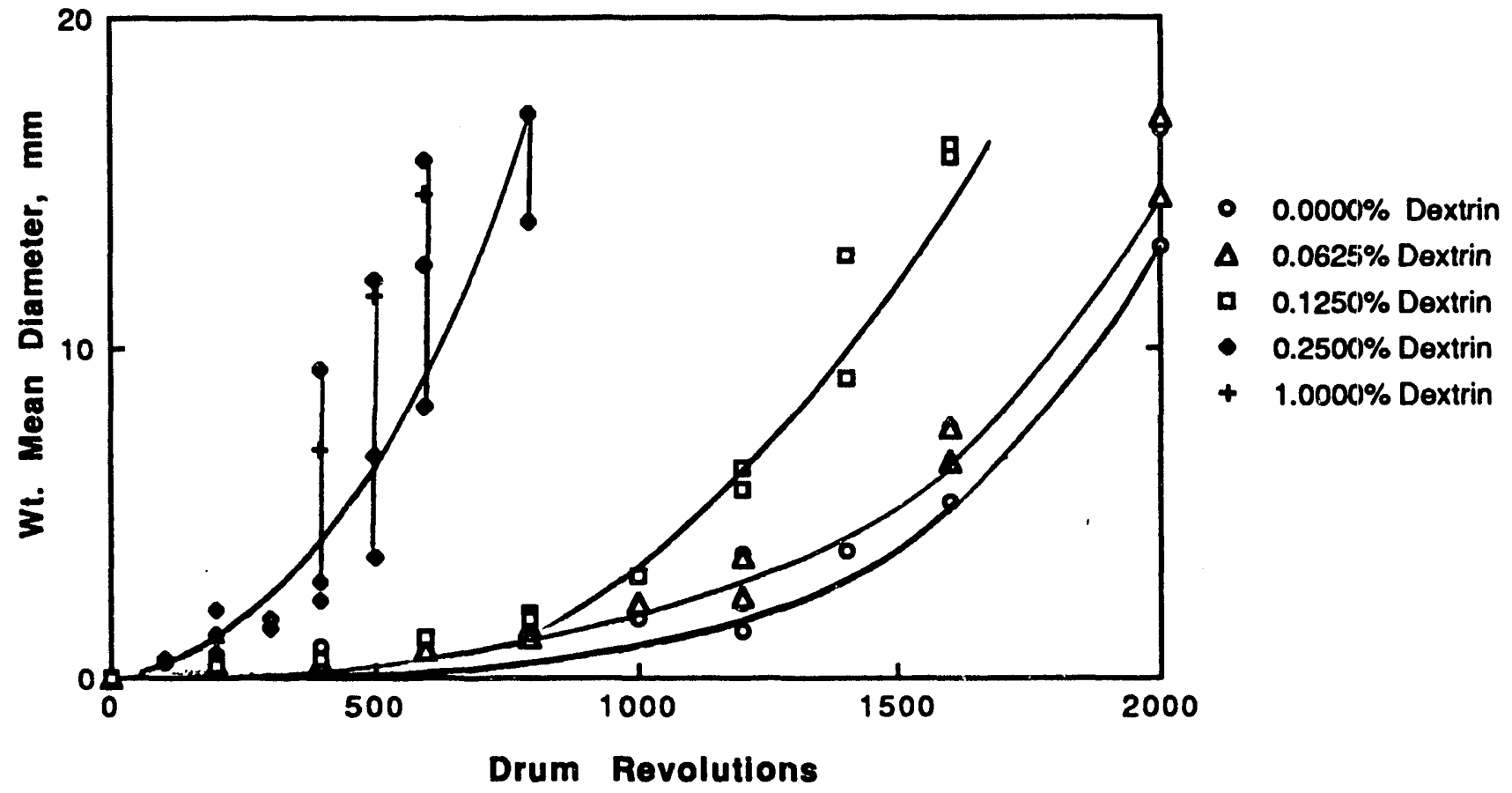

Figure 2. Influence of dextrin on the batch pelletization kinetics of Upper Freeport coal. 


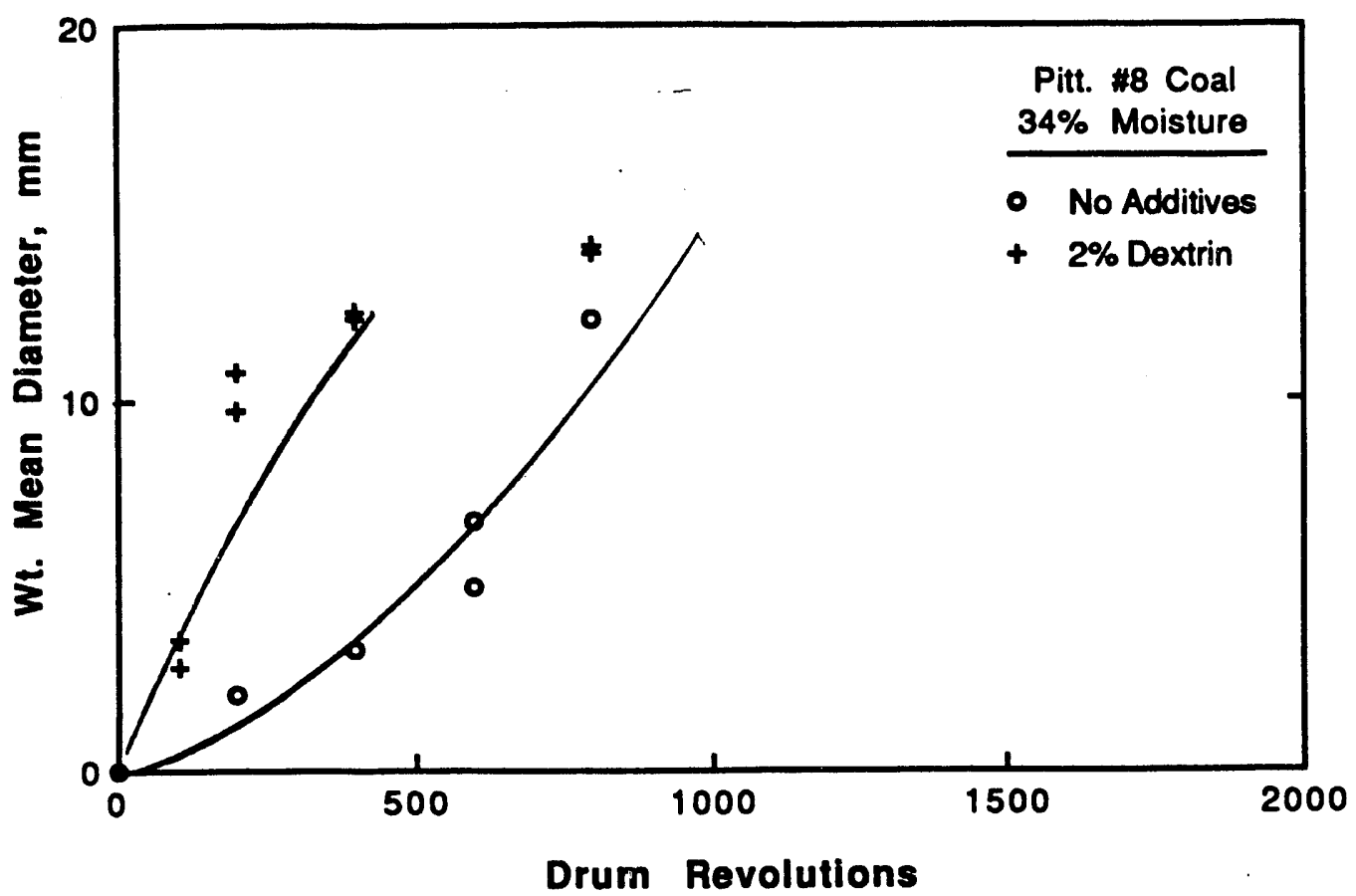

Figure 3. Influence of dextrin on the batch pelletization kinetics of Pittsburgh $\# 8$ coal.

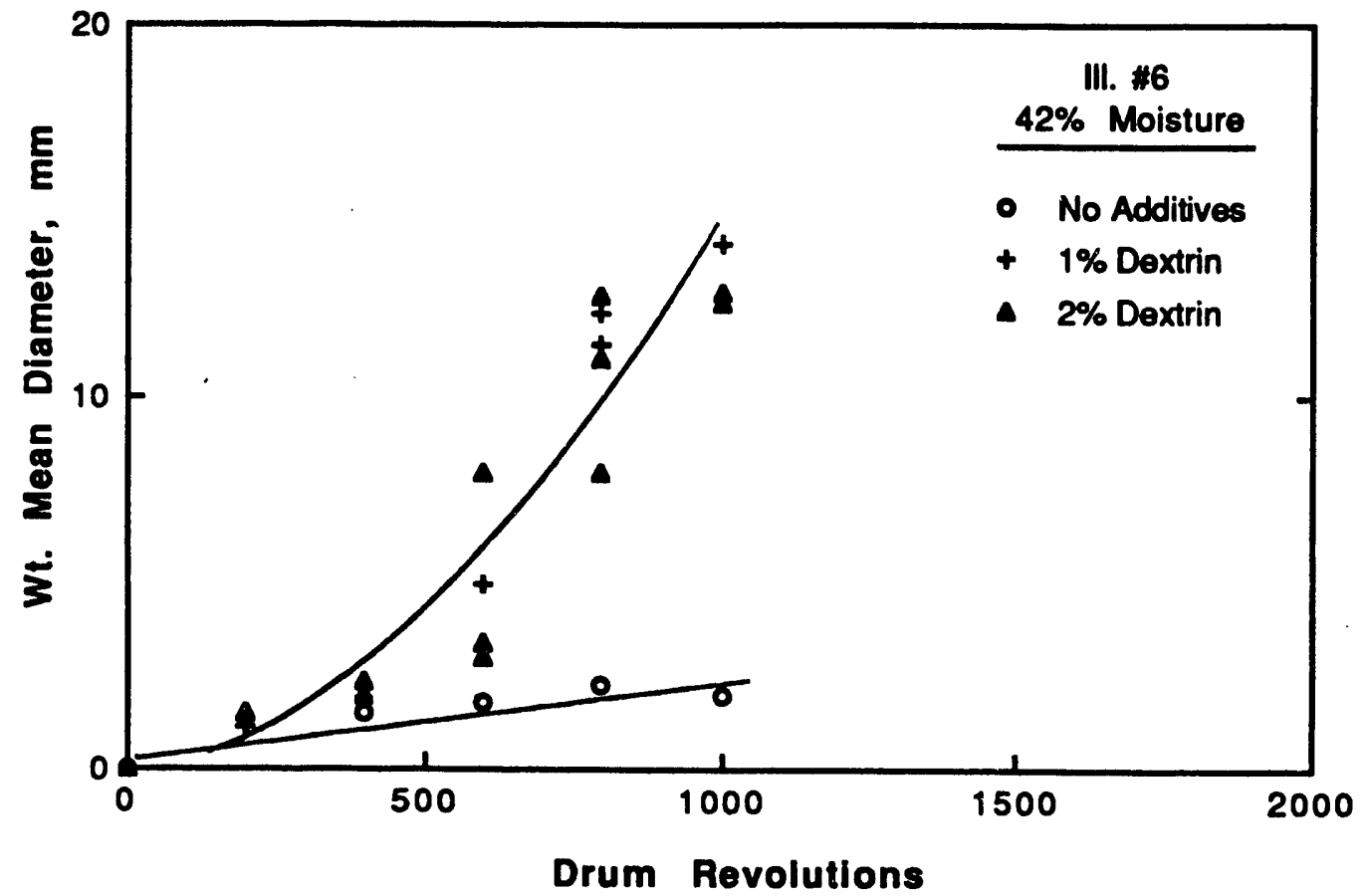

Figure 4. Influence of dextrin on the batch pelletization kinetics of Illinois \#6 coal. 


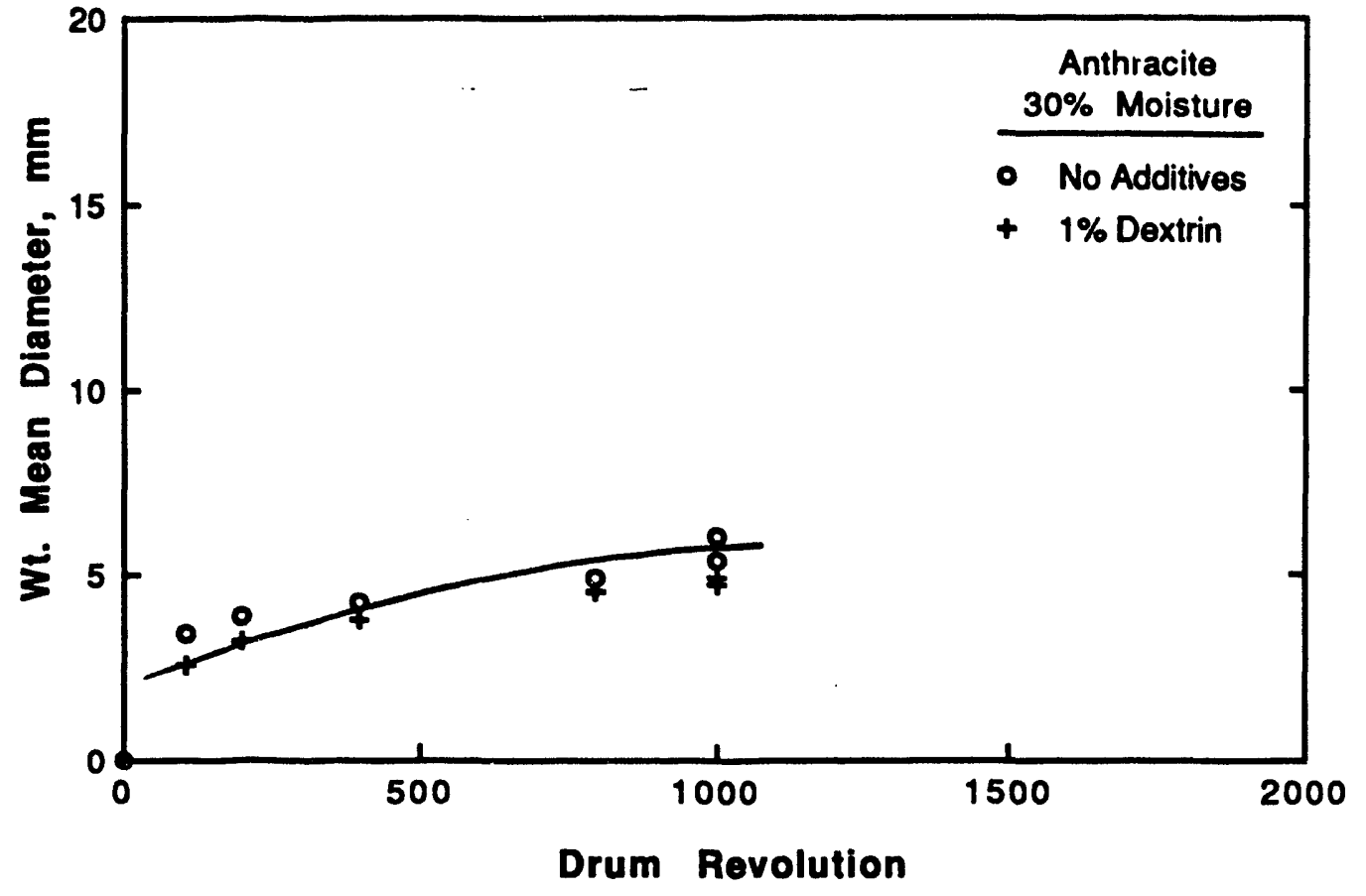

Figure 5. Influence of dextrin on the batch pelletization kinetics of anthracite. 

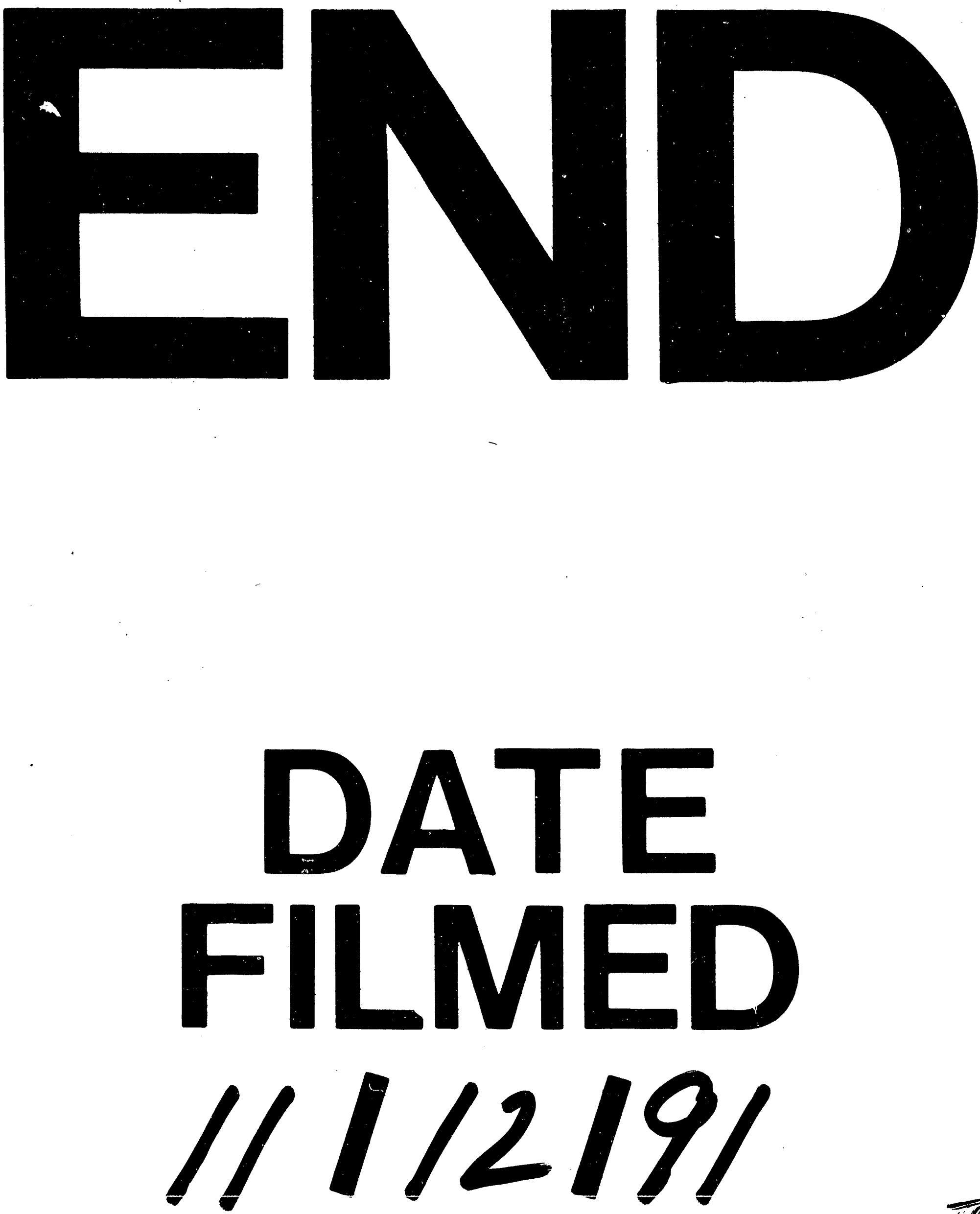
